\title{
On $\gamma$-vectors satisfying the Kruskal-Katona inequalities
}

\author{
E. Nevo $\|^{\text {tand T. K. Petersen }}{ }^{2}$ \\ ${ }^{1}$ Department of Mathematics, Cornell University, Ithaca USA \\ ${ }^{2}$ Department of Mathematical Sciences, DePaul University, Chicago USA
}

\begin{abstract}
We present examples of flag homology spheres whose $\gamma$-vectors satisfy the Kruskal-Katona inequalities. This includes several families of well-studied simplicial complexes, including Coxeter complexes and the simplicial complexes dual to the associahedron and to the cyclohedron. In these cases, we construct explicit flag simplicial complexes whose $f$-vectors are the $\gamma$-vectors in question, and so a result of Frohmader shows that the $\gamma$-vectors satisfy not only the Kruskal-Katona inequalities but also the stronger Frankl-Füredi-Kalai inequalities. In another direction, we show that if a flag $(d-1)$-sphere has at most $2 d+3$ vertices its $\gamma$-vector satisfies the Frankl-FürediKalai inequalities. We conjecture that if $\Delta$ is a flag homology sphere then $\gamma(\Delta)$ satisfies the Kruskal-Katona, and further, the Frankl-Füredi-Kalai inequalities. This conjecture is a significant refinement of Gal's conjecture, which asserts that such $\gamma$-vectors are nonnegative.

Résumé. Nous présentons des exemples de sphères d'homologie flag dont $\gamma$-vecteurs satisfaire les inégalités de Kruskal-Katona. Cela comprend plusieurs familles de bien étudiés simplicial complexes, y compris les complexes de Coxeter et les complexes simpliciaux dual de l'associahedron et à la cyclohedron. Dans ces cas, nous construisons explicite flag simplicial complexes dont $f$-vecteurs sont les $\gamma$-vecteurs en question, et ainsi de suite de Frohmader montre que le $\gamma$-vecteurs de satisfaire non seulement les inégalités de Kruskal-Katona mais aussi la plus fortes inégalités Frankl-Füredi-Kalai. Dans une autre direction, nous montrons que, si un flag $(d-1)$-sphère a au plus $2 d+3$ ses sommets $\gamma$-vecteur satisfait aux inégalités de Frankl-Füredi-Kalai. Nous conjecture que, si $\Delta$ est une sphère d'homologie flag alors $\gamma(\Delta)$ satisfait aux inégalités de Kruskal-Katona, en outre, les de Frankl-Füredi-Kalai. Cette conjecture est un raffinement significative de la conjecture de Gal, qui affirme que ces $\gamma$-vecteurs sont nonnégatifs.
\end{abstract}

Keywords: simplicial complex, Coxeter complex, associahedron, Gal's conjecture, $\gamma$-vector

\section{Introduction}

In [Ga] Gal gave counterexamples to the real-root conjecture for flag spheres and conjectured a weaker statement which still implies the Charney-Davis conjecture. The conjecture is phrased in terms of the so-called $\gamma$-vector.

Conjecture 1.1 (Gal) $G a$ Conjecture 2.1.7] If $\Delta$ is a flag homology sphere then $\gamma(\Delta)$ is nonnegative.

\footnotetext{
${ }^{\dagger}$ Partially supported by NSF Award DMS-0757828

1365-8050 @ 2010 Discrete Mathematics and Theoretical Computer Science (DMTCS), Nancy, France
} 
This conjecture is known to hold for the order complex of a Gorenstein* poset [Kar], all Coxeter complexes (see [Ste], and references therein), and for the (dual simplicial complexes of the) "chordal nestohedra" of [PoRWi] - a class containing the associahedron, permutahedron, and other well-studied polytopes.

If $\Delta$ has a nonnegative $\gamma$-vector, one may ask what these nonnegative integers count. In certain cases (the type A Coxeter complex, say), the $\gamma$-vector has a very explicit combinatorial description. We will exploit such descriptions to show that not only are these numbers nonnegative, but they satisfy certain non-trivial inequalities known as the Kruskal-Katona inequalities. Put another way, such a $\gamma$-vector is the $f$-vector of a simplicial complex. Our main result is the following.

Theorem 1.2 The $\gamma$-vector of $\Delta$ satisfies the Kruskal-Katona inequalities for each of the following classes of flag spheres:

(a) $\Delta$ is a Coxeter complex.

(b) $\Delta$ is the simplicial complex dual to an associahedron.

(c) $\Delta$ is the simplicial complex dual to a cyclohedron (type B associahedron).

Note that the type A Coxeter complex is dual to the permutahedron, and for types B and D there is a similarly defined polytope-the "Coxeterhedron" of Reiner and Ziegler [RZ].

We prove Theorem 1.2 by constructing, for each such $\Delta$, a simplicial complex whose faces correspond to the combinatorial objects enumerated by $\gamma(\Delta)$.

In a different direction, we are also able to show that if $\Delta$ is a flag sphere with few vertices relative to its dimension, then its $\gamma$-vector satisfies the Kruskal-Katona inequalities.

Theorem 1.3 Let $\Delta$ be a $(d-1)$-dimensional flag homology sphere with at most $2 d+3$ vertices, i.e., with $\gamma_{1}(\Delta) \leq 3$. Then $\gamma(\Delta)$ satisfies the Kruskal-Katona inequalities. Moreover, all possible $\gamma$-polynomials with $\gamma_{1} \leq 3$ that satisfy the Kruskal-Katona inequalities, except for $1+3 t+3 t^{2}$, occur as $\gamma(\Delta ; t)$ for some flag sphere $\Delta$.

The proof of Theorem 1.3 can be found in [NPe]. It characterizes the structure of such flag spheres.

Computer evidence suggests that Theorems 1.2 and 1.3 may be enlarged significantly. We make the following strengthening of Gal's conjecture.

Conjecture 1.4 If $\Delta$ is a flag homology sphere then $\gamma(\Delta)$ satisfies the Kruskal-Katona inequalities.

This conjecture is true, but not sharp, for flag homology 3- (or 4-) spheres. Indeed, Gal showed that $0 \leq \gamma_{2}(\Delta) \leq \gamma_{1}(\Delta)^{2} / 4$ must hold for flag homology 3- (or 4-) spheres [Ga], which implies the Kruskal-

Katona inequality $\gamma_{2}(\Delta) \leq\left(\begin{array}{c}\gamma_{1}(\Delta) \\ 2\end{array}\right)$. Our stronger Conjecture 5.3 is sharp for flag homology spheres of dimension at most 4 .

In Section 2 we review some key definitions. Section 3 collects some known results describing the combinatorial objects enumerated by the $\gamma$-vectors of Theorem 1.2. Section 4 constructs simplicial complexes based on these combinatorial objects and proves Theorem 1.2 Finally, Section 5 describes a strengthening of Theorem 1.2 by showing that under the same hypotheses the stronger Frankl-Füredi-Kalai inequalities hold for the $\gamma$-vector. A stronger companion to Conjecture 1.4 is also presented, namely Conjecture 5.3

This paper is an abridged version of [NPe]. Full definitions and proofs can be found there. 


\section{Terminology}

We assume the reader has a basic familiarity with abstract simplicial complexes.

We say that $\Delta$ is flag if all the minimal subsets of $V$ which are not in $\Delta$ have size 2; equivalently $F \in \Delta$ if and only if all the edges of $F$ (two element subsets) are in $\Delta$.

The $f$-polynomial of a $(d-1)$-dimensional simplicial complex $\Delta$ is the generating function for the dimensions of the faces of the complex:

$$
f(\Delta ; t):=\sum_{F \in \Delta} t^{\operatorname{dim} F+1}=\sum_{0 \leq i \leq d} f_{i}(\Delta) t^{i}
$$

The $f$-vector

$$
f(\Delta):=\left(f_{0}, f_{1}, \ldots, f_{d}\right)
$$

is the sequence of coefficients of the $f$-polynomial.

The $h$-polynomial of $\Delta$ is a transformation of the $f$-polynomial:

$$
h(\Delta ; t):=(1-t)^{d} f(\Delta ; t /(1-t))=\sum_{0 \leq i \leq d} h_{i}(\Delta) t^{i},
$$

and the $h$-vector is the corresponding sequence of coefficients,

$$
h(\Delta):=\left(h_{0}, h_{1}, \ldots, h_{d}\right) .
$$

Though they contain the same information, often the $h$-polynomial is easier to work with than the $f$ polynomial. For instance, if $\Delta$ is a homology sphere, then the Dehn-Sommerville relations guarantee that the $h$-vector is symmetric, i.e., $h_{i}=h_{d-i}$ for all $0 \leq i \leq d$.

When referring to the $f$ - or $h$-polynomial of a simple polytope, we mean the $f$-or $h$-polynomial of the boundary complex of its dual. So, for instance, we refer to the $h$-vector of the type A Coxeter complex and the permutahedron interchangeably.

Whenever a polynomial of degree $d$ has symmetric integer coefficients, it has an integer expansion in the basis $\left\{t^{i}(1+t)^{d-2 i}: 0 \leq i \leq d / 2\right\}$. Specifically, if $\Delta$ is a $(d-1)$-dimensional homology sphere then there exist integers $\gamma_{i}(\Delta)$ such that

$$
h(\Delta ; t)=\sum_{0 \leq i \leq d / 2} \gamma_{i}(\Delta) t^{i}(1+t)^{d-2 i} .
$$

We refer to the sequence $\gamma(\Delta):=\left(\gamma_{0}, \gamma_{1}, \ldots\right)$ as the $\gamma$-vector of $\Delta$, and the corresponding generating function $\gamma(\Delta ; t)=\sum \gamma_{i} t^{i}$ is the $\gamma$-polynomial. Our goal is to show that under the hypotheses of Theorems 1.2 and 1.3 the $\gamma$-vector for $\Delta$ is seen to be the $f$-vector for some other simplicial complex.

A result of Schützenberger, Kruskal and Katona (all independently), characterizes the $f$-vectors of simplicial complexes. (See [Sta, Ch. II.2].) By convention we call the conditions characterizing these $f$-vectors the Kruskal-Katona inequalities.

We will use the Kruskal-Katona inequalities directly for Theorem 1.3 and for checking the Coxeter complexes of exceptional type in part (a) of Theorem 1.2. (See Table 1) For the remainder of Theorem 1.2 we construct explicit simplicial complexes with the desired $f$-vectors. 


\begin{tabular}{c|c}
$W$ & $\gamma(W)$ \\
\hline \hline$E_{6}$ & $(1,1266,7104,3104)$ \\
\hline$E_{7}$ & $(1,17628,221808,282176)$ \\
\hline$E_{8}$ & $(1,881744,23045856,63613184,17111296)$ \\
\hline$F_{4}$ & $(1,232,208)$ \\
\hline$G_{2}$ & $(1,8)$ \\
\hline$H_{3}$ & $(1,56)$ \\
\hline$H_{4}$ & $(1,2632,3856)$ \\
\hline$I_{2}(m)$ & $(1,2 m-4)$ \\
\hline
\end{tabular}

Tab. 1: The $\gamma$-vectors for finite Coxeter complexes of exceptional type.

\section{Combinatorial descriptions of $\gamma$-nonnegativity}

Here we provide combinatorial descriptions (mostly already known) for the $\gamma$-vectors of the complexes described in Theorem 1.2 .

\subsection{Type A Coxeter complex}

We begin by describing the combinatorial objects enumerated by the $\gamma$-vector of the type $A_{n-1}$ Coxeter complex, or equivalently, the permutahedron. (For the reader looking for more background on the Coxeter complex itself, we refer to [H, Section 1.15]; for the permutahedron see [Z, Example 0.10].)

Recall that a descent of a permutation $w=w_{1} w_{2} \cdots w_{n} \in \mathfrak{S}_{n}$ is a position $i \in[n-1]$ such that $w_{i}>w_{i+1}$. A peak (resp. valley) is a position $i \in[2, n-1]$ such that $w_{i-1}<w_{i}>w_{i+1}$ (resp. $\left.w_{i-1}>w_{i}<w_{i+1}\right)$. We let des(w) denote the number of descents of $w$, and we let peak $(w)$ denote the number of peaks. It is well known that the $h$-polynomial of the type $A_{n-1}$ Coxeter complex is expressed as:

$$
h\left(A_{n-1} ; t\right)=\sum_{w \in \mathfrak{S}_{n}} t^{\operatorname{des}(w)} .
$$

Foata and Schützenberger were the first to demonstrate the $\gamma$-nonnegativity of this polynomial (better known as the Eulerian polynomial), showing $h\left(A_{n-1} ; t\right)=\sum \gamma_{i} t^{i}(1+t)^{n-1-2 i}$, where $\gamma_{i}=$ the number of equivalence classes of permutations of $n$ with $i+1$ peaks [FoSch]. (Two permutations are in the same equivalence class if they have the same sequence of values at their peaks and valleys.) See also Shapiro, Woan, and Getu [ShWoGe] and, in a broader context, Brändén $[\bar{B}]$ and Stembridge [Ste].

Following Postnikov, Reiner, and Williams [PoRWi], we choose the following set of representatives for these classes:

$$
\widehat{\mathfrak{S}}_{n}=\left\{w \in \mathfrak{S}_{n}: w_{n-1}<w_{n}, \text { and if } w_{i-1}>w_{i} \text { then } w_{i}<w_{i+1}\right\}
$$

In other words, $\widehat{\mathfrak{S}}_{n}$ is the set of permutations $w$ with no double descents and no final descent, or those for which $\operatorname{des}(w)=\operatorname{peak}(0 w 0)-1$. We now phrase the $\gamma$-nonnegativity of the type $A_{n-1}$ Coxeter complex in this language. 
Theorem 3.1 (Foata-Schützenberger) [FoSch Théorème 5.6] The h-polynomial of the type $A_{n-1}$ Coxeter complex can be expressed as follows:

$$
h\left(A_{n-1} ; t\right)=\sum_{w \in \widehat{\mathfrak{S}}_{n}} t^{\operatorname{des}(w)}(1+t)^{n-1-2 \operatorname{des}(w)} .
$$

We now can state precisely that the type $A_{n-1}$ Coxeter complex (permutahedron) has $\gamma\left(A_{n-1}\right)=$ $\left(\gamma_{0}, \gamma_{1}, \ldots, \gamma_{\left\lfloor\frac{n-1}{2}\right\rfloor}\right)$, where

$$
\gamma_{i}\left(A_{n-1}\right)=\left|\left\{w \in \widehat{\mathfrak{S}}_{n}: \operatorname{des}(w)=i\right\}\right| .
$$

The permutahedron is an example of a chordal nestohedron. Following [PoRWi], a chordal nestohedron $P_{\mathcal{B}}$ is characterized by its building set, $\mathcal{B}$. Each building set $\mathcal{B}$ on $[n]$ has associated to it a set of $\mathcal{B}$ permutations, $\mathfrak{S}_{n}(\mathcal{B}) \subset \mathfrak{S}_{n}$, and we similarly define $\widehat{\mathfrak{S}}_{n}(\mathcal{B})=\mathfrak{S}_{n}(\mathcal{B}) \cap \widehat{\mathfrak{S}}_{n}$. See [PoRWi] for details. The following is a main result of Postnikov, Reiner, and Williams [PoRWi].

Theorem 3.2 (Postnikov, Reiner, Williams) [PoRWi Theorem 11.6] If $\mathcal{B}$ is a connected chordal building set on $[n]$, then

$$
h\left(P_{\mathcal{B}} ; t\right)=\sum_{w \in \widehat{\mathfrak{S}}_{n}(\mathcal{B})} t^{\operatorname{des}(w)}(1+t)^{n-1-2 \operatorname{des}(w)} .
$$

Thus, for a chordal nestohedron, $\gamma_{i}\left(P_{\mathcal{B}}\right)=\left|\left\{w \in \widehat{\mathfrak{S}}_{n}(\mathcal{B}): \operatorname{des}(w)=i\right\}\right|$.

\subsection{Type B Coxeter complex}

We now turn our attention to the type $B_{n}$ Coxeter complex. The framework of [PoRWi] no longer applies, so we must discuss a new, if similar, combinatorial model.

In type $B_{n}$, the $\gamma$-vector is given by $\gamma_{i}=4^{i}$ times the number of permutations $w$ of $\mathfrak{S}_{n}$ such that $\operatorname{peak}(0 w)=i$. See Petersen $[\overline{\mathrm{Pe}}]$ and Stembridge [Ste]. We define the set of decorated permutations $D e c_{n}$ as follows. A decorated permutation $\mathbf{w} \in D e c_{n}$ is a permutation $w \in \mathfrak{S}_{n}$ with bars following the peak positions (with $w_{0}=0$ ). Moreover these bars come in four colors: $\left\{\left.|=|\right|^{0},\left.\right|^{1},\left.\right|^{2},\left.\right|^{3}\right\}$. Thus for each $w \in \mathfrak{S}_{n}$ we have $4^{\text {peak }(0 w)}$ decorated permutations in $D e c_{n}$. For example, $D e c_{9}$ includes elements such as

$$
4|238|^{1} 76519,\left.\left.\quad 4\right|^{3} 238\right|^{2} 76519,\left.\quad 25|137|^{1} 69\right|^{2} 84 .
$$

(Note that $\widehat{\mathfrak{S}}_{n} \subset D e c_{n}$.) Let peak $(\mathbf{w})=\operatorname{peak}(0 w)$ denote the number of bars in $\mathbf{w}$. In this context we have the following result.

Theorem 3.3 (Petersen) [Pe] Proposition 4.15] The h-polynomial of the type $B_{n}$ Coxeter complex can be expressed as follows:

$$
h\left(B_{n} ; t\right)=\sum_{\mathbf{w} \in \operatorname{Dec} n} t^{\text {peak }(\mathbf{w})}(1+t)^{n-2 p e a k(\mathbf{w})}
$$

Thus,

$$
\gamma_{i}\left(B_{n}\right)=\left|\left\{\mathbf{w} \in \operatorname{Dec}_{n}: \operatorname{peak}(\mathbf{w})=i\right\}\right|
$$




\subsection{Type D Coxeter complex}

We now describe how to view the elements enumerated by the $\gamma$-vector of the type D Coxeter complex in terms of a subset of decorated permutations. Define a subset $D e c_{n}^{D} \subset D e c_{n}$ as follows:

$$
\begin{array}{r}
D e c_{n}^{D}=\left\{\mathbf{w}=\left.w_{1} \cdots\left|{ }^{c_{1}} w_{i_{1}} \cdots\right|\right|^{c_{2}} \cdots \in D e c_{n} \text { such that } w_{1}<w_{2}<w_{3},\right. \text { or, } \\
\text { both } \left.\max \left\{w_{1}, w_{2}, w_{3}\right\} \neq w_{3} \text { and } c_{1} \in\{0,1\}\right\} .
\end{array}
$$

In other words, we remove from $D e c_{n}$ all elements whose underlying permutations have $w_{2}<w_{1}<w_{3}$, then for what remains we dictate that bars in the first or second positions can only come in one of two colors. Stembridge [Ste] gives an expression for the $h$-polynomial of the type $D_{n}$ Coxeter complex, which we now phrase in the following manner.

Theorem 3.4 (Stembridge) [Ste Corollary A.5]. The h-polynomial of the type $D_{n}$ Coxeter complex can be expressed as follows:

$$
h\left(D_{n} ; t\right)=\sum_{\mathbf{w} \in D e c_{n}^{D}} t^{p e a k(\mathbf{w})}(1+t)^{n-2 p e a k(\mathbf{w})} .
$$

Thus,

$$
\gamma_{i}\left(D_{n}\right)=\left|\left\{\mathbf{w} \in \operatorname{Dec}_{n}^{D}: \operatorname{peak}(\mathbf{w})=i\right\}\right| .
$$

\subsection{The associahedron}

The associahedron $A s s o c_{n}$ is an example of a chordal nestohedron, so Theorem 3.2 applies. Following [PoRWi, Section 10.2], the $\mathcal{B}$-permutations of $A s s o c_{n}$ are precisely the 312 -avoiding permutations. Let $\mathfrak{S}_{n}(312)$ denote the set of all $w \in \mathfrak{S}_{n}$ such that there is no triple $i<j<k$ with $w_{j}<w_{k}<w_{i}$. Then we have:

$$
h\left(\text { Assoc }_{n} ; t\right)=\sum_{w \in \widehat{\mathfrak{S}}_{n}(312)} t^{\operatorname{des}(w)}(1+t)^{n-1-2 \operatorname{des}(w)},
$$

where $\widehat{\mathfrak{S}}_{n}(312)=\mathfrak{S}_{n}(312) \cap \widehat{\mathfrak{S}}_{n}$. Hence,

$$
\gamma_{i}\left(\operatorname{Assoc}_{n}\right)=\left|\left\{w \in \widehat{\mathfrak{S}}_{n}(312): \operatorname{des}(w)=i\right\}\right| .
$$

\subsection{The cyclohedron}

The cyclohedron $C y c_{n}$, or type B associahedron, is a nestohedron, though not a chordal nestohedron and hence Theorem 3.2 does not apply. Its $\gamma$-vector can be explicitly computed from its $h$-vector as described in [PoRWi, Proposition 11.15]. We have $\gamma_{i}\left(C y c_{n}\right)=\left(\begin{array}{c}n \\ i, i, n-2 i\end{array}\right)$. Define

$$
P_{n}=\{(L, R) \subseteq[n] \times[n]:|L|=|R|, L \cap R=\emptyset\} .
$$

It is helpful to think of elements of $P_{n}$ as follows. For $\sigma=(L, R)$ with $|L|=|R|=k$, write $\sigma$ as a $k \times 2$ array with the elements of $L$ written in increasing order in the first column, the elements of $R$ in increasing order in the second column. That is, if $L=\left\{l_{1}<\cdots<l_{k}\right\}$ and $R=\left\{r_{1}<\cdots<r_{k}\right\}$, we write

$$
\sigma=\left(\begin{array}{cc}
l_{1} & r_{1} \\
\vdots & \vdots \\
l_{k} & r_{k}
\end{array}\right) .
$$


For $\sigma \in P_{n}$, let $\rho(\sigma)=|L|=|R|$. Then we can write

$$
h\left(C y c_{n} ; t\right)=\sum_{\sigma \in P_{n}} t^{\rho(\sigma)}(1+t)^{n-2 \rho(\sigma)} .
$$

Thus,

$$
\gamma_{i}\left(C y c_{n}\right)=\left|\left\{\sigma \in P_{n}: \rho(\sigma)=i\right\}\right|
$$

\section{The $\Gamma$-complexes}

We will now describe simplicial complexes whose $f$-vectors are the $\gamma$-vectors described in Section 3 .

\subsection{Coxeter complexes}

Notice that if

$$
\mathbf{w}=\left.\left.w_{1}\right|^{c_{1}} \ldots\left|{ }^{c_{i-1}} w_{i}\right|^{c_{i}} w_{i+1}\right|^{c_{i+1}} \ldots||^{c_{l-1}} w_{l},
$$

is a decorated permutation, then each word $w_{i}=w_{i, 1} \ldots w_{i, k}$ has some $j$ such that:

$$
w_{i, 1}>w_{i, 2}>\cdots>w_{i, j}>w_{i, j+1}<w_{i, j+2}<\cdots<w_{i, k}
$$

We say $w_{i}$ is a down-up word. We call $\grave{w}_{i}=w_{i, 1} \cdots w_{i, j}$ the decreasing part of $w_{i}$ and $\dot{w}_{i}=w_{i, j+1} \cdots w_{i, k}$ the increasing part of $w_{i}$. Note that the decreasing part may be empty, whereas the increasing part is nonempty if $i \neq l$. Also, the rightmost block of $\mathbf{w}$ may be strictly decreasing (in which case $w_{l}=\grave{w}_{l}$ ) and the leftmost block is always increasing, even if it is a singleton.

Define the vertex set

$$
V_{D e c_{n}}:=\left\{\mathbf{v} \in \operatorname{Dec} c_{n}: \operatorname{peak}(\mathbf{v})=1\right\} .
$$

The adjacency of two such vertices is defined as follows. Let

$$
\mathbf{u}=\left.\dot{u}_{1}\right|^{c} \grave{u}_{2} \dot{u}_{2}
$$

and

$$
\mathbf{v}=\left.\dot{v}_{1}\right|^{d} \grave{v}_{2} \dot{v}_{2}
$$

be two vertices with $\left|\dot{u}_{1}\right|<\left|\hat{v}_{1}\right|$. We define $\mathbf{u}$ and $\mathbf{v}$ to be adjacent if and only if there is an element $\mathbf{w} \in D e c_{n}$ such that

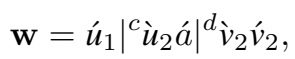

where $a$ is the letters of $\dot{u}_{2} \cap \dot{v}_{1}$ written in increasing order. Such an element $\mathbf{w}$ exists if, as sets:

- $\dot{u}_{1} \cup \grave{u}_{2} \subset \dot{v}_{1}\left(\Leftrightarrow \grave{v}_{2} \cup \dot{v}_{2} \subset \dot{u}_{2}\right)$,

- $\min \dot{u}_{2} \cap \dot{v}_{1}<\min \grave{u}_{2}$, and

- $\max \dot{u}_{2} \cap \dot{v}_{1}>\max \grave{v}_{2}$. (Note that $\dot{u}_{2} \cap \dot{v}_{1}$ is nonempty by the first condition.)

Definition 4.1 Let $\Gamma\left(D e c_{n}\right)$ be the collection of all subsets $F$ of $V_{D e c_{n}}$ such that every two distinct vertices in $F$ are adjacent. 
Note that by definition $\Gamma\left(D e c_{n}\right)$ is a flag complex. It remains to show that the faces of $\Gamma\left(D e c_{n}\right)$ correspond to decorated permutations.

Let $\phi: D e c_{n} \rightarrow \Gamma\left(D e c_{n}\right)$ be the map defined as follows. If

$$
\mathbf{w}=\left.\left.\left.\left.w_{1}\right|^{c_{1}} \cdots||^{c_{i-1}} w_{i}\right|^{c_{i}} w_{i+1}\right|^{c_{i+1}} \cdots\right|^{c_{l-1}} w_{l},
$$

then

$$
\phi(\mathbf{w})=\left\{\left.w_{1}\right|^{c_{1}} \grave{w}_{2} \dot{b}_{1}, \ldots,\left.\dot{a}_{i}\right|^{c_{i}} \grave{w}_{i+1} \dot{b}_{i}, \ldots,\left.\dot{a}_{l-1}\right|^{c_{l-1}} \grave{w}_{l} \dot{b}_{l-1}\right\},
$$

where $\dot{a}_{i}$ is the set of letters to the left of $\grave{w}_{i+1}$ in $\mathbf{w}$ written in increasing order and $\hat{b}_{i}$ is the set of letters to the right of $\grave{w}_{i+1}$ in $\mathbf{w}$ written in increasing order.

Proposition 4.2 The map $\phi$ is a bijection between faces of $\Gamma\left(D_{e} c_{n}\right)$ and decorated permutations in Dec ${ }_{n}$.

The proof of Proposition 4.2 is not difficult; it can be found in [NPe].

We now make explicit how to realize $D e c_{n}$ as the face poset of $\Gamma\left(D e c_{n}\right)$. We say $\mathbf{w}$ covers $\mathbf{u}$ if and only if $\mathbf{u}$ can be obtained from $\mathbf{w}$ by removing a bar $\left.\right|^{c_{i}}$ and reordering the word $w_{i} w_{i+1}=\grave{w}_{i} \dot{w}_{i} w_{i+1}$ as a down-up word $\grave{w}_{i} a$ where $a$ is the word formed by writing the letters of $\dot{w}_{i} w_{i+1}$ in increasing order. Then $\left(D e c_{n}, \leq\right)$ is a poset graded by number of bars and we have the following result.

Proposition 4.3 The map $\phi$ is an isomorphism of graded posets from $\left(\operatorname{Dec}_{n}, \leq\right)$ to $\left(\Gamma\left(D_{e c}\right), \subseteq\right)$.

We now claim that the $\gamma$-objects for the type $A_{n-1}$ and type $D_{n}$ Coxeter complexes form flag subcomplexes of $\Gamma\left(D e c_{n}\right)$. Let $S \in\left\{\widehat{\mathfrak{S}}_{n}, D e c_{n}^{D}\right\}$. To show $\Gamma(S)$ is a subcomplex, by Proposition 4.3 it suffices to show that $(S, \leq)$ is a lower ideal in $\left(D e c_{n}, \leq\right)$. To show that $\Gamma(S)$ is flag, we show that it is the flag complex generated by the elements of $S$ with exactly one bar. Both facts are straightforward to verify for either choice of $S$.

Proposition 4.4 For $S \in\left\{\widehat{\mathfrak{S}}_{n}, D e c_{n}^{D}\right\}$ the image $\Gamma(S):=\phi(S)$ is a flag subcomplex of $\Gamma\left(D e c_{n}\right)$.

In light of the results of Sections 3.1 3.2, and 3.3. and because the dimension of faces corresponds to the number of bars, we have the following result, which, along with Table 1 implies part (a) of Theorem 1.2 .

Corollary 4.5 We have:

1. $\gamma\left(A_{n-1}\right)=f\left(\Gamma\left(\widehat{\mathfrak{S}}_{n}\right)\right)$,

2. $\gamma\left(B_{n}\right)=f\left(\Gamma\left(D e c_{n}\right)\right.$, and

3. $\gamma\left(D_{n}\right)=f\left(\Gamma\left(D e c_{n}^{D}\right)\right.$.

In particular, the $\gamma$-vectors of the type $A_{n-1}, B_{n}$, and $D_{n}$ Coxeter complexes satisfy the Kruskal-Katona inequalities.

Remark 4.6 In view of Theorem 3.2 we can observe that if $\mathcal{B}$ is a connected chordal building set such that $\left(\widehat{\mathfrak{S}}_{n}(\mathcal{B}), \leq\right)$ is a lower ideal in $\left(D e c_{n}, \leq\right)$, then a result such as Corollary 4.5 applies. That is, we would have $\gamma\left(P_{\mathcal{B}}\right)=f\left(\phi\left(\widehat{\mathfrak{S}}_{n}(\mathcal{B})\right)\right)$. In particular, we would like to apply such an approach to the $\gamma$ vector of the associahedron. However, $\widehat{\mathfrak{S}}_{n}(312)$ is not generally a lower ideal in Dec ${ }_{n}$. For example, with $n=5$, we have $w=3|14| 25>3 \mid 1245=u$. While $w$ is 312-avoiding, $u$ is clearly not. 


\subsection{The associahedron}

First we give a useful characterization of the set $\widehat{\mathfrak{S}}_{n}(312)$.

Observation 4.7 If $w \in \widehat{\mathfrak{S}}_{n}(312)$, it has the form

$$
w=a_{1} j_{1} i_{1} \dot{a}_{2} j_{2} i_{2} \cdots a_{k} j_{k} i_{k} a_{k+1},
$$

where:

- $j_{1}<\cdots<j_{k}$,

- $j_{s}>i_{s}$ for all $s$, and

- $\dot{a}_{s}$ is the word formed by the letters of $\left\{r \in[n] \backslash\left\{i_{1}, j_{1}, \ldots, i_{k}, j_{k}\right\}: j_{s-1}<r<j_{s}\right\}$ (with $j_{0}=0$, $\left.j_{k+1}=n+1\right)$ written in increasing order.

In particular, since $w$ has no double descents and no final descent, we see that $a_{k+1}$ is always nonempty and $w_{n}=n$. We refer to $\left(i_{s}, j_{s}\right)$ as a descent pair of $w$.

Given distinct integers $a, b, c, d$ with $a<b$ and $c<d$, we say the pairs $(a, b)$ and $(c, d)$ are crossing if either of the following statements are true:

- $a<c<b<d$ or

- $c<a<d<b$.

Otherwise, we say the pairs are noncrossing. For example, $(1,5)$ and $(4,7)$ are crossing, whereas both the pairs $(1,5)$ and $(2,4)$ and the pairs $(1,5)$ and $(6,7)$ are noncrossing.

Define the vertex set

$$
V_{\widehat{\mathfrak{S}}_{n}(312)}:=\{(a, b): 1 \leq a<b \leq n-1\} .
$$

Definition 4.8 Let $\Gamma\left(\widehat{\mathfrak{S}}_{n}(312)\right)$ be the collection of subsets $F$ of $V_{\widehat{\mathfrak{S}}_{n}(312)}$ such that every two distinct vertices in $F$ are noncrossing.

By definition $\Gamma\left(\widehat{\mathfrak{S}}_{n}(312)\right)$ is a flag simplicial complex, and so the task remains to show that the faces of the complex correspond to the elements of $\widehat{\mathfrak{S}}_{n}(312)$.

Define a map $\pi: \widehat{\mathfrak{S}}_{n}(312) \rightarrow \Gamma\left(\widehat{\mathfrak{S}}_{n}(312)\right)$ as follows:

$$
\pi(w)=\left\{\left(w_{i+1}, w_{i}\right): w_{i}>w_{i+1}\right\} .
$$

Suppose $w$ is as in (1). We claim that the descent pairs $\left(i_{s}, j_{s}\right)$ and $\left(i_{t}, j_{t}\right)$ (with $j_{s}<j_{t}$, say) are noncrossing. Indeed, if $i_{s}<i_{t}<j_{s}<j_{t}$, then the subword $j_{s} i_{s} i_{t}$ forms the pattern 312. Therefore (and because $w_{n}=n$ ) we see the map $\pi(w)=\left\{\left(i_{1}, j_{1}\right), \ldots,\left(i_{k}, j_{k}\right)\right\}$ is well-defined. Using Observation 4.7. the following is straightforward to prove.

Proposition 4.9 The map $\pi$ is a bijection between faces of $\Gamma\left(\widehat{\mathfrak{S}}_{n}(312)\right)$ and $\widehat{\mathfrak{S}}_{n}(312)$.

By construction, we have $|\pi(w)|=\operatorname{des}(w)$, and therefore the results of Section 3.4 imply the following result, proving part (b) of Theorem 1.2 . 
Corollary 4.10 We have:

$$
\gamma\left(\operatorname{Assoc}_{n}\right)=f\left(\Gamma\left(\widehat{\mathfrak{S}}_{n}(312)\right)\right) .
$$

In particular, the $\gamma$-vector of the associahedron satisfies the Kruskal-Katona inequalities.

Remark 4.11 It is well known that the h-vector of the associahedron has a combinatorial interpretation given by noncrossing partitions. Simion and Ullmann [SiU] give a particular decomposition of the lattice of noncrossing partitions that can be used to describe $\gamma\left(\right.$ Assoc $\left._{n}\right)$ in (essentially) the same way.

\subsection{The cyclohedron}

For the cyclohedron, let

$$
V_{P_{n}}:=\{(l, r) \in[n] \times[n]: l \neq r\} .
$$

Two vertices $\left(l_{1}, r_{1}\right)$ and $\left(l_{2}, r_{2}\right)$ are adjacent if and only if:

- $l_{1}, l_{2}, r_{1}, r_{2}$ are distinct and

- $l_{1}<l_{2}$ if and only if $r_{1}<r_{2}$.

Define $\Gamma\left(P_{n}\right)$ to be the flag complex whose faces $F$ are all subsets of $V_{P_{n}}$ such that every two distinct vertices in $F$ are adjacent.

We let $\psi: P_{n} \rightarrow \Gamma\left(P_{n}\right)$ be defined as follows. If

$$
\sigma=\left(\begin{array}{cc}
l_{1} & r_{1} \\
\vdots & \vdots \\
l_{k} & r_{k}
\end{array}\right)
$$

is an element of $P_{n}$, then $\psi(\sigma)$ is simply the set of rows of $\sigma$ :

$$
\psi(\sigma)=\left\{\left(l_{1}, r_{1}\right), \ldots,\left(l_{k}, r_{k}\right)\right\} .
$$

Clearly this map is invertible, for we can list a set of pairwise adjacent vertices in increasing order (by $l_{i}$ or by $r_{i}$ ) to obtain an element of $P_{n}$. We have the following.

Proposition 4.12 The map $\psi$ is a bijection between faces of $\Gamma\left(P_{n}\right)$ and the elements of $P_{n}$.

We are now able to complete the proof of Theorem 1.2 as the following implies part (c).

Corollary 4.13 We have

$$
\gamma\left(\operatorname{Cyc}_{n}\right)=f\left(\Gamma\left(P_{n}\right)\right) .
$$

In particular, the $\gamma$-vector of the cyclohedron satisfies the Kruskal-Katona inequalities. 


\section{Stronger inequalities}

A $(d-1)$-dimensional simplicial complex $\Delta$ on a vertex set $V$ is balanced if there is a coloring of its vertices $c: V \rightarrow[d]$ such that for every face $F \in \Delta$ the restriction map $c: F \rightarrow[d]$ is injective. That is, every face has distinctly colored vertices.

Frohmader [Fro] proved that the $f$-vectors of flag complexes form a (proper) subset of the $f$-vectors of balanced complexes. (This was conjectured earlier by Eckhoff and Kalai, independently.) Further, a characterization of the $f$-vectors of balanced complexes is known [FraFüKal], yielding stronger upper bounds on $f_{i+1}$ in terms of $f_{i}$ than the Kruskal-Katona inequalities, namely the Frankl-Füredi-Kalai inequalities. For example, a balanced 1-dimensional complex is a bipartite graph, hence satisfies $f_{2} \leq$ $f_{1}^{2} / 4$, while the complete graph has $f_{2}=\left(\begin{array}{c}f_{1} \\ 2\end{array}\right)$. See [FraFüKal] for the general description of the FranklFüredi-Kalai inequalities.

Because the $\Gamma$-complexes of Section 4 are flag complexes, Frohmader's result shows that the $\gamma$-vectors of Theorem 1.2 satisfy the Frankl-Füredi-Kalai inequalities. The same is easily verified for the $\gamma$-vectors given by Theorem 1.3 and in Table 1 for the exceptional Coxeter complexes. We obtain the following strengthening of Theorem 1.2 .

Theorem 5.1 The $\gamma$-vector of $\Delta$ satisfies the Frankl-Füredi-Kalai inequalities for each of the following classes of flag spheres:

(a) $\Delta$ is a Coxeter complex.

(b) $\Delta$ is the simplicial complex dual to an associahedron.

(c) $\Delta$ is the simplicial complex dual to a cyclohedron.

(d) $\Delta$ has $\gamma_{1}(\Delta) \leq 3$

Remark 5.2 The complexes $\Gamma(S)$ where $S \in\left\{D e c_{n}, \widehat{\mathfrak{S}}_{n}, D e c_{n}^{D}\right\}$ are balanced. The color of a vertex $v$ with a peak at position $i$ is $\left\lceil\frac{i}{2}\right\rceil$.

Similarly this suggests the following strengthening of Conjecture 1.4

Conjecture 5.3 If $\Delta$ is a flag homology sphere then $\gamma(\Delta)$ satisfies the Frankl-Füredi-Kalai inequalities.

As mentioned in the Introduction, this conjecture is true for flag homology spheres of dimension at most 4 . We do not have a counterexample to the following possible strengthening of this conjecture.

Problem 5.4 If $\Delta$ is a flag homology sphere, then $\gamma(\Delta)$ is the $f$-vector of a flag complex.

\section{References}

[B] P. Brändén, Sign-graded posets, unimodality of $W$-polynomials and the Charney-Davis conjecture, Electron. J. Combin. 11 (2004/06), Research Paper 9, 15pp.

[FraFüKal] P. Frankl, Z. Füredi and G. Kalai, Shadows of colored complexes, Math. Scand. 63 (1988), 169-178.

[Fro] A. Frohmader, Face vectors of flag complexes, Israel J. Math. 164 (2008), 153-164. 
[FoSch] D. Foata and M.-P. Schützenberger, “Théorie géométrique des polynômes eulériens," Lecture Notes in Mathematics, Vol. 138, Springer-Verlag, Berlin, 1970.

[Ga] S. R. Gal, Real root conjecture fails for five- and higher-dimensional spheres, Discrete Comput. Geom. 34 (2005), 269-284.

$[\mathrm{H}] \quad$ J. E. Humphreys, "Reflection groups and Coxeter groups," Cambridge Univ. Press, Cambridge, 1990.

[Kar] K. Karu, The $c d$-index of fans and posets, Compos. Math. 142 (2006), 701-718.

[M] R. Meshulam, Domination numbers and homology, J. Combin. Theory Ser. A 102 (2003), $321-330$.

[N] E. Nevo, Higher minors and Van Kampen's obstruction, Math. Scand. 101 (2007), 161176.

[NPe] E. Nevo and T. K. Petersen, On $\gamma$-vectors satisfying the Kruskal-Katona inequalities, arXiv: 0909.0694.

[Pe] T. K. Petersen, Enriched P-partitions and peak algebras, Adv. Math. 209 (2007), 561-610.

[PoRWi] A. Postnikov, V. Reiner, and L. Williams, Faces of generalized permutohedra, Doc. Math. 13 (2008) 207-273.

[RZ] V. Reiner and G. Ziegler, Coxeter-associahedra, Mathematika 41 (1994), 364-393.

[ShWoGe] L. W. Shapiro, W. J. Woan, S. Getu, Runs, slides and moments, SIAM J. Algebraic Discrete Methods 4 (1983), 459-466.

[SiU] R. Simion and D. Ullman, On the structure of the lattice of noncrossing partitions, Discrete Math. 98 (1991), 193-206.

[Sta] R. P. Stanley, "Combinatorics and Commutative Algebra" (2nd ed.), Birkhäuser, Boston, 1996.

[Ste] J. R. Stembridge, Coxeter cones and their $h$-vectors, Adv. Math. 217 (2008) 1935-1961.

[Z] G. M. Ziegler, "Lectures on polytopes," Graduate Texts in Mathematics, 152, SpringerVerlag, New York, 1995. 